\title{
Long-Term Survival After Salvage Thoracic Surgery on a Patient with ALK-Rearranged Metastatic Lung Adenocarcinoma After Progression on Targeted Therapy
}

\author{
Kangqi Ren ${ }^{1, *}$ \\ Guanggui Ding ${ }^{1, *}$ \\ Shuying Xie $\mathbb{B}^{2}$ \\ Lin Yang (D) ${ }^{\prime}$
}

'Department of Thoracic Surgery, Shenzhen People's Hospital \& The Second Clinical Medical College of Jinan University, Shenzhen, People's Republic of China; ${ }^{2}$ Department of Medicine, Burning Rock Biotech, Guangzhou, People's Republic of China

*These authors contributed equally to this work
Correspondence: Lin Yang

Department of Thoracic Surgery,

Shenzhen People's Hospital \& The Second

Clinical Medical College of Jinan

University, 1017 North Dongmen Road,

Shenzhen, People's Republic of China

Tel +86-137983। 4779

Email szrmyyxwk10@163.com

\begin{abstract}
Targeted therapy for patients with advanced non-small cell lung cancer (NSCLC) is often challenged by the arising of drug resistance. After progression to targeted therapy, treatment options include continued targeted therapy, definitive local therapy, and the combination of both. While there is evidence that local ablative radiotherapy may prolong the disease control by targeted therapy, little is known regarding the relevance of salvage thoracic surgery in this setting. Herein, we presented a case of stage IV lung adenocarcinoma with concurrent $E M L 4-A L K$ and $T A C 1-A L K$ fusion who had long-term survival after salvage thoracic surgery. The patient underwent a multidisciplinary treatment scheme that consisted of radiotherapy, ALK inhibitor crizotinib, and surgery, with blood-based genomic profiling for monitoring disease progression. Notably, salvage thoracic surgery was performed after progression on the crizotinib therapy and acquired $A L K$ F1174C mutation was identified, which has been shown to be resistant to crizotinib and possibly sensitive to ceritinib. The patient benefited from salvage thoracic surgery with a remarkable progression-free survival of 31 months at last follow-up, and the patient maintained high-performance status throughout the course of management. To the best of our knowledge, this is the first case reporting on the long-term survival outcome from salvage thoracic surgery after crizotinib treatment in an NSCLC patient carrying double $A L K$ fusion.
\end{abstract}

Keywords: advanced non-small cell lung cancer, multidisciplinary treatment, salvage thoracic surgery, crizotinib, $A L K$ F1174C mutation

\section{Introduction}

Non-small cell lung cancer (NSCLC) makes up nearly $85 \%$ of lung cancer cases and is one of the most lethal forms of malignancies. Surgery is currently the cornerstone of NSCLC treatment since it provides an opportunity for cure. Unfortunately, more than $70 \%$ of patients are diagnosed with advanced NSCLC (stage III and IV) at diagnosis. The standard-of-care consists largely of systemic treatment modalities, such as concomitant chemo- and radiotherapy for the majority of patients in stage III, and targeted therapy, chemotherapy, immunotherapy, or chemo-immunotherapy for stage IV patients and some stage III NSCLC patients. However, salvage thoracic surgery may still be a viable option for advanced NSCLC (aNSCLC) beyond frontline treatment, as the disease progression as a result of drug resistance. ${ }^{1}$ Salvage surgery of lung tumors refers to the resection of residual or recurrent tumors or in urgencies 
such as severe infections. There is evidence that tumor excision, indicated by disease downstaging to resectable tumor or improved performance status after targeted therapy, may be safe and beneficial. ${ }^{2}$ However, in the setting of progression on targeted therapy, although definitive local therapies, such as local ablative radiotherapy and surgery, are recommended for subsequent treatment, the role of salvage thoracic surgery is poorly characterized. A recent retrospective analysis of the relevance of surgical resection after treatment with epidermal growth factor receptor (EGFR) or anaplastic lymphoma kinase (ALK) inhibitors reported a median recurrence-free survival of 15 months after salvage thoracic surgery. ${ }^{2}$ However, the majority of patients (33/36) harbored EGFR alterations, and few (3/36) patients carried $A L K$ fusions in this previous study. ${ }^{2}$ Therefore, there is a dearth of evidence in assessing the safety and efficacy of salvage thoracic surgery in $A L K$-altered, progressive aNSCLC. Herein, we described the multidisciplinary treatment and outcome of a stage IV lung adenocarcinoma patient harboring double $A L K$ fusion. Surgery was performed to remove the enlarged primary lung tumor after second-line therapy with crizotinib. At the time of manuscript preparation, the patient had no evidence of recurrence or progression with a progression-free survival (PFS) of 31 months.

\section{Case Presentation}

A 55-year-old woman was referred to our hospital in Nov 2016 with a two-month history of left-sided hip pain. Chest computed tomography (CT) noted a mass in the left lower pulmonary lobe $(6.0 \mathrm{~cm}$, Figure 1A). Pelvic CT revealed a soft tissue mass with bone destruction, which suggested a neoplastic lesion in the left-hip joint (Figure 1D and G). A needle biopsy of the primary lung tumor was conducted, and a pathologic review led to a diagnosis of stage IV lung adenocarcinoma (T2aNxM1b). As there was insufficient material for mutational profiling of the lung biopsy, next-generation sequencing (NGS) with a panel of 168 lung cancer-related genes (Burning Rock Biotech, Guangzhou, China) was performed with plasma and identified two $A L K$ fusions, $E M L 4-A L K$ (E6:A20) fusion with a mutational allele frequency (AF) of $5.47 \%$ and $T A C 1-A L K$ (Tintergenic: A20) fusion with an AF of 5.27\%. The patient was then started on a combination of crizotinib, zoledronic acid, and left hip joint-directed radiotherapy in Dec 2016.

Dynamic surveillance with plasma-based NGS in Feb 2017 revealed that either $A L K$ fusions or other previously identified genomic aberrations were detected, and circulating tumor DNA (ctDNA) clearance was observed in all subsequent tests (Figure 2). Follow-up CT scans in Oct 2017 showed a reduction in the primary lung lesion $(2.0 \mathrm{~cm})$ consistent with a response evaluation of partial response. The enlargement of the primary lung lesion (Figure 1B) and stabilization of the left hip joint lesion was noted in Jan 2018 (Figure 1E). The patient was subsequently given a revised regimen with crizotinib, zoledronic acid, and bevacizumab based on the following reasons. First, at the time of progression on first-line treatment (Jan 2018), no standard-of-care was recommended in guidelines of Chinese Society of Clinical Oncology (CSCO, http://www. csco.org.cn/cn/index.aspx) for the treatment of patients with $A L K$-rearranged metastatic NSCLC who were previously treated with crizotinib and data on the efficacy of novel ALK-tyrosine kinase inhibitors (TKIs) in a randomized clinical trial is lack. Second, it has been documented that patient with advanced $A L K$-rearranged NSCLC after initial disease progression could obtain benefit from continued crizotinib. ${ }^{3}$ Third, several clinical trial studies have demonstrated that bevacizumab, as a recombinant humanized monoclonal IgG1 antibody that binds to and inhibits the biologic activity of human vascular endothelial growth factor (VEGF), is safe, feasible, and effective as frontline treatment of advanced NSCLC. ${ }^{4,5}$ Given the local progression after treatment of crizotinib and radiotherapy plus zoledronic acid, crizotinib beyond progression combined with bevacizumab and zoledronic acid were given to the patient between Jan 2018 and Jul 2018. Positron emission tomography (PET)-CT scans in Apr 2018 revealed increased metabolic activity in both the primary lung tumor (Figure 1H) and metastatic lesion to the left-hip joint (Figure 1I).

Despite the initial partial response, the disease progressed six months later, when CT scans showed an enlarged primary lung tumor (Figure 1C) while the hip joint metastasis remained stable (Figure 1F). After multidisciplinary consultation and preoperative evaluation, the patient was evaluated at an Eastern Cooperative Oncology Group performance status (ECOG PS) of 1 and deemed eligible for surgery. Video-assisted thoracoscopic left lower lobectomy and systematic nodal dissection were performed. Gross total resection of the primary lung tumor with a diameter of $2.9 \times 3.6 \mathrm{~cm}$ was achieved. Pathologic review of surgical specimens revealed a left lung adenocarcinoma with no necrosis, metastases to the 7th and 11th group of lymph nodes, and no metastases to the 6th, 9th and 10th group of lymph nodes. NGS profiling of the primary lung tumor with a panel of 520 cancer-related genes (Burning 

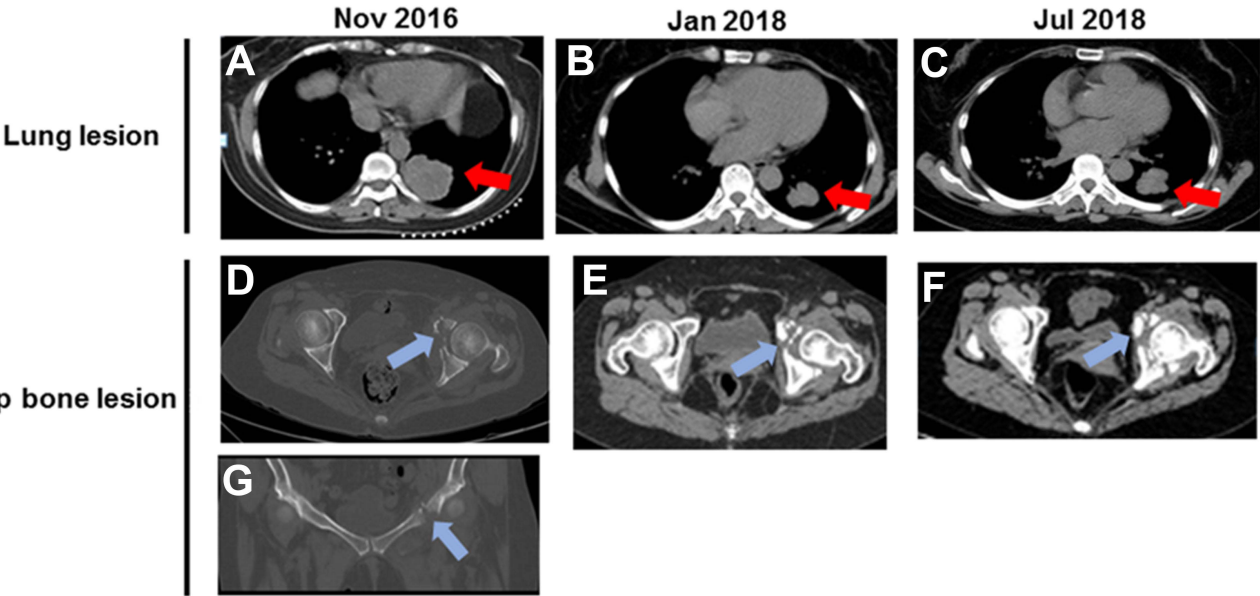

H

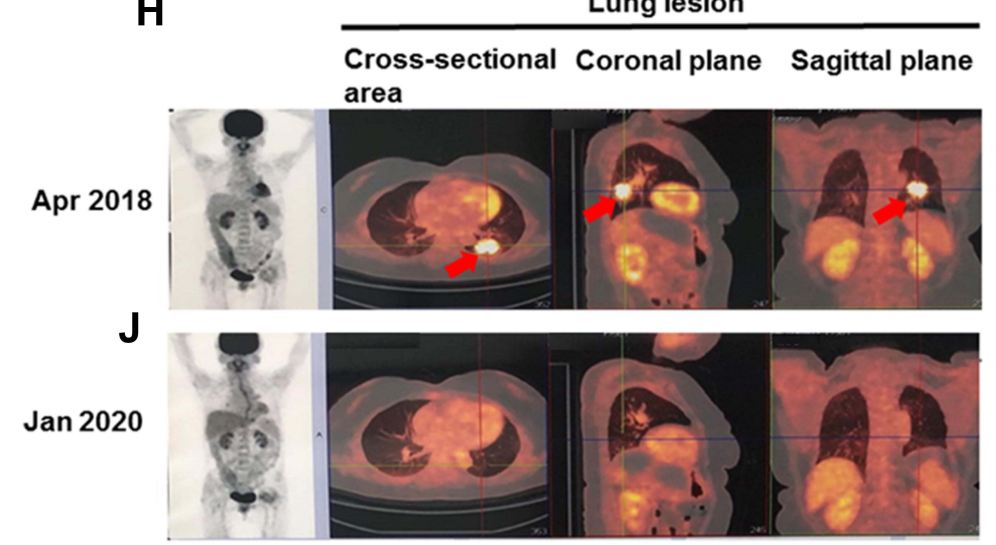

I Cross-sectional Coronal plane Sagittal plane
area
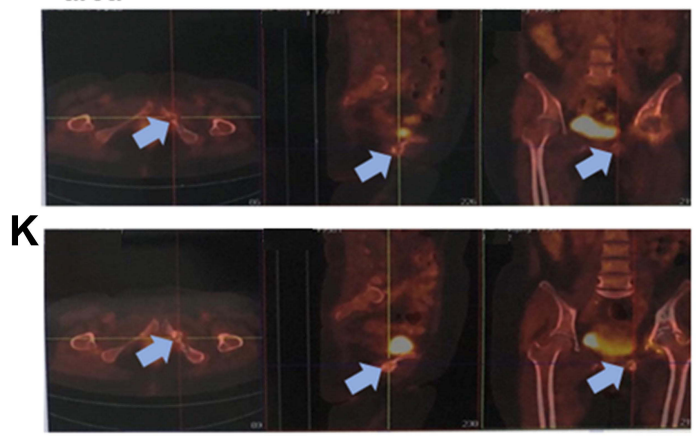

Figure I PET-CT/CT scans of the primary lung tumor and hip bone lesion. Chest CT scans of the primary lung tumor prior to first-line treatment (A), after first-line treatment (B), and after second-line treatment (C); Pelvis CT scans of the hip bone lesion prior to first-line treatment (D and $\mathbf{G})$, after first-line treatment (E), and after second-line treatment (F); PET-CT scans of the primary lung tumor in Apr 2018 (H) and Jan 2020 (J); PET-CT scans of the metastatic lesion to left hip joint in Apr 2018 (I) and Jan 2020 (K). Red arrows indicated the primary lung tumors and blue arrows indicated the hip bone lesions.

Abbreviation: PET-CT, positron emission tomography-computerized tomography.

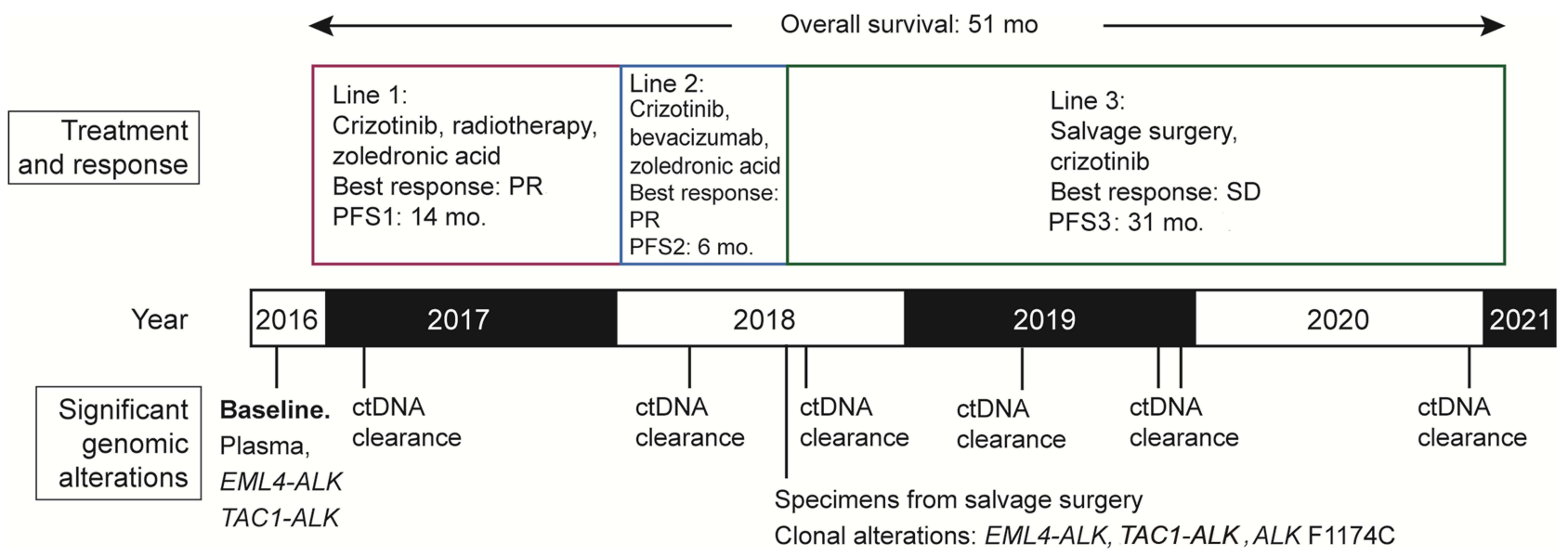

Figure 2 Diagram presentation of multimodal treatment described in this report. The patient underwent three lines of therapies that consisted of radiotherapy, ALK inhibitor crizotinib, and surgery, with molecular monitoring. Notably, salvage thoracic surgery was performed after progression on second-line treatment based on crizotinib and was followed by a remarkable PFS of 31 months at last follow-up.

Abbreviations: ctDNA, circulating tumor DNA; PR, partial response; PFS, progression-free survival; SD, stable disease. 
Rock Biotech, Guangzhou, China) revealed three clonal alterations: $A L K$ F1174C with an AF of 9.39\%, EML4$A L K$ fusion with an $\mathrm{AF}$ of $16.05 \%$, and $T A C 1-A L K$ (Tintergenic: A20) fusion with an AF of $15.51 \%$. Crizotinib was continued after the surgery. As of the follow-up in Jan 2020, there was no sign of recurrent disease in the lung (Figure 1J), and the bone metastasis remained stable (Figure 1K). The patient was in high-performance status (ECOG PS of 1). She had therefore achieved an overall survival of 51 months, including a noteworthy 31month PFS after salvage thoracic surgery (Figure 2).

\section{Discussion}

In this report, we presented a case of successful salvage thoracic surgery following progression on targeted therapy in a metastatic, $A L K$-rearranged lung adenocarcinoma patient. The role of salvage thoracic surgery in treating aNSCLC has not been well characterized. In the selected advanced EGFR-mutant NSCLC patients who received EGFR inhibitor gefitinib and then achieved a resectable disease, removal of residual disease was associated with a median overall survival of 31 months, but recurrencefree survival after surgery was unsatisfactory. ${ }^{6}$ For patients progressing on targeted therapy, local treatment modalities are recommended. Although local ablative therapy has been shown to prolong the duration of disease control when combined with continuation of the EGFR- or ALK-TKIs (median PFS of six months), little is known regarding the safety and benefit of surgical resection in this setting. ${ }^{7}$ Our case therefore provides valuable evidence that salvage thoracic surgery after progression on ALKTKIs may lead to favorable survival outcomes.

Although $A L K$ rearrangements have been reported in $5 \%$ to $6 \%$ of NSCLC patients, more than 30 types of $A L K$ fusion partners (such as $E M L 4, K I F 5 B$, and $K L C 1$ ) have been identified in NSCLC to date. ${ }^{8}$ Double $A L K$ fusion is a very rare event in $A L K$-rearranged NSCLCs. To the best of our knowledge, only a few of cases have been documented in the literature, which reveal that patients with NSCLC who harbor double $A L K$ fusion (such as NLRC4$A L K$ and EML4-ALK, CDK15-ALK and EML4-ALK, $C C N Y-A L K$ and $A T I C-A L K, P R K C B-A L K$ and EML4$A L K, B C L 11 A-A L K$ and $E M L 4-A L K)$ could benefit from ALK-TKI crizotinib. ${ }^{9-13}$ In the present work, the patient is the first reported case that harbors concurrent TAC1-ALK (Tintergenic: A20) and $E M L 4-A L K$ fusion. We also found that this patient achieved durable response to crizotinib plus radiotherapy with a PFS of 14 months. Our findings provide important information for future treatment decision-making in patients with double $A L K$ fusion.

Moreover, compared with other local treatment options, surgery offers a unique advantage as it could enable genomic profiling of the tumor tissue and therefore guilding treatment options.${ }^{14}$ Dynamic surveillance with NGS is routinely performed with blood for most aNSCLC patients on targeted therapy, and in the usual case when neoplasms driven by TKI-resistant mutations emerge and eventually manifest as progressive disease, these mutations are more likely to be identified with tissue-based testing. ${ }^{15}$ This advantage is illustrated in our case, in which all three surgical specimens were found to harbor an additional $A L K \mathrm{~F} 1174 \mathrm{C}$, a mutation known to render resistance to crizotinib and perhaps sensitive to ceritinib based upon structural insights and sporadic reports. ${ }^{16}$ An in silico study has revealed the allosteric effect of F1174C resistance mutation on ceritinib in ALK. ${ }^{16}$ An in vitro study has demonstrated that $A L K \mathrm{~F} 1174 \mathrm{C}$ is resistant to crizotinib and sensitive to ceritinib, alectinib, brigatinib, and lolatinib. ${ }^{17}$ In addition, alectinib, brigatinib, and lolatinib have been approved for the treatment of patients with metastatic $A L K$-positive NSCLC. These findings suggest that patients harboring $A L K \mathrm{~F} 1174 \mathrm{C}$ might benefit from alectinib, brigatinib or lolatinib. Clinical trials are needed to explore the efficacies of these three ALK-TKIs in metastatic $A L K$-rearranged NSCLC patients who acquired resistance mutation $A L K \quad \mathrm{~F} 1174 \mathrm{C}$ after crizotinib treatment.

In summary, we described the successful multimodal treatment of a metastatic lung adenocarcinoma patient carrying double $A L K$ fusion at diagnosis. The course of management spanned over 51 months, consisting of three lines of therapies that comprised targeted therapy and salvage thoracic surgery. Notably, the excision of the residual lung tumor was followed by a PFS of 31 months. To the best of our knowledge, this is the first case report on the long-term survival outcome after salvage thoracic surgery as a later-line treatment for advanced NSCLC patients with double $A L K$ fusion.

\section{Ethics Statement}

This study was approved by the Ethics Committee of Shenzhen People's Hospital. Patient provided informed consent to the study and permitted the use of tumor tissue. Written informed consent was obtained from the patient for publication of this case report. An institutional approval has been obtained to publish the case details. 


\section{Disclosure}

The authors report no conflicts of interest in this work.

\section{References}

1. Kim C, Hoang CD, Kesarwala AH, Schrump DS, Guha U, Rajan A Role of local ablative therapy in patients with oligometastatic and oligoprogressive non-small cell lung cancer. $J$ Thorac Oncol. 2017;12:179-193. doi:10.1016/j.jtho.2016.10.012

2. Ohtaki Y, Shimizu K, Suzuki H, et al. Salvage surgery for non-small cell lung cancer after tyrosine kinase inhibitor treatment. Lung Cancer. 2021;153:108-116. doi:10.1016/j.lungcan.2020.12.037

3. Hong X, Chen Q, Ding L, et al. Clinical benefit of continuing crizotinib therapy after initial disease progression in Chinese patients with advanced ALK-rearranged non-small-cell lung cancer. Oncotarget. 2017;8:41631-41640. doi:10.18632/oncotarget.15892

4. Kim ES, Moon J, Herbst RS, et al. Phase II trial of carboplatin, paclitaxel, cetuximab, and bevacizumab followed by cetuximab and bevacizumab in advanced nonsquamous non-small-cell lung cancer: SWOG S0536. J Thorac Oncol. 2013;8:1519-1528. doi:10.1097/ JTO.0000000000000009

5. Schneider BJ, Kalemkerian GP, Gadgeel SM, et al. Phase II trial of dose-dense pemetrexed, gemcitabine, and bevacizumab in patients with advanced, non-small-cell lung cancer. Clin Lung Cancer. 2017;18:299-302. doi:10.1016/j.cllc.2016.11.019

6. Song W, Di S, Liu J, et al. Salvage surgery for advanced non-small cell lung cancer after targeted therapy: a case series. Thorac Cancer. 2020;11:1061-1067. doi:10.1111/1759-7714.13366

7. Weickhardt AJ, Rothman MS, Salian-Mehta S, et al. Rapid-onset hypogonadism secondary to crizotinib use in men with metastatic nonsmall cell lung cancer. Cancer. 2012;118:5302-5309. doi:10.1002/cncr.27450

8. Friedlaender A, Banna G, Patel S, Addeo A. Diagnosis and treatment of ALK aberrations in metastatic NSCLC. Curr Treat Options Oncol. 2019;20:79. doi:10.1007/s11864-019-0675-9
9. Wu X, Wang W, Zou B, et al. Novel NLRC4-ALK and EML4-ALK double fusion mutations in a lung adenocarcinoma patient: a case report. Thorac Cancer. 2020;11:1695-1698. doi:10.1111/17597714.13389

10. Guo J, Shi J, Yao M, et al. A rare double ALK fusion variant EML4-ALK and CDK15-ALK in lung adenocarcinoma and response to crizotinib: a case report. Medicine. 2020;99:e22631. doi:10.1097/ MD.0000000000022631

11. Wu X, Zhou H, He Z, et al. Coexistence of a novel CCNY-ALK and ATIC-ALK double-fusion in one patient with ALK-positive NSCLC and response to crizotinib: a case report. Transl Lung Cancer Res. 2020;9:2494-2499. doi:10.21037/tlcr-20-1049

12. Luo J, Gu D, Lu H, Liu S, Kong J. Coexistence of a novel PRKCB-ALK, EML4-ALK double-fusion in a lung adenocarcinoma patient and response to crizotinib. J Thorac Oncol. 2019;14:e266e268. doi:10.1016/j.jtho.2019.07.021

13. Qin BD, Jiao XD, Liu K, Wu Y, Zang YS. Identification of a novel EML4-ALK, BCL11A-ALK double-fusion variant in lung adenocarcinoma using next-generation sequencing and response to crizotinib. J Thorac Oncol. 2019;14:e115-e117. doi:10.1016/j.jtho.2019.01.032

14. Zhang C, Wang L, Li W, et al. Surgical outcomes of stage IV non-small cell lung cancer: a single-center experience. J Thorac Dis. 2019;11:5463-5473. doi:10.21037/jtd.2019.11.30

15. Weickhardt AJ, Doebele RC, Camidge DR. A time to test, a time to treat. J Thorac Dis. 2012;4:223-225.

16. Ni Z, Wang X, Zhang T, Jin RZ. Molecular dynamics simulations reveal the allosteric effect of $\mathrm{F} 1174 \mathrm{C}$ resistance mutation to ceritinib in ALK-associated lung cancer. Comput Biol Chem. 2016;65:54-60. doi:10.1016/j.compbiolchem.2016.10.005

17. Gainor JF, Dardaei L, Yoda S, et al. Molecular mechanisms of resistance to first- and second-generation ALK inhibitors in ALK-rearranged lung cancer. Cancer Discov. 2016;6:1118-1133. doi:10.1158/2159-8290.CD-16-0596
OncoTargets and Therapy

\section{Publish your work in this journal}

OncoTargets and Therapy is an international, peer-reviewed, open access journal focusing on the pathological basis of all cancers, potential targets for therapy and treatment protocols employed to improve the management of cancer patients. The journal also focuses on the impact of management programs and new therapeutic

Submit your manuscript here: https://www.dovepress.com/oncotargets-and-therapy-journal

\section{Dovepress}

agents and protocols on patient perspectives such as quality of life, adherence and satisfaction. The manuscript management system is completely online and includes a very quick and fair peer-review system, which is all easy to use. Visit http://www.dovepress.com/ testimonials.php to read real quotes from published authors. 